\title{
Habilidades lingüísticas en el desarrollo de la capacidad investigativa
}

\author{
Linguistic skills in the development of research capacity
}

\author{
Fidelia Nina Luna \\ fidelianina24@gmail.com

\begin{abstract}
Resumen
El objetivo del desarrollo de la capacidad investigativa en los estudiantes, es preparar para el ejercicio de una ciudadanía activa y consciente de la biodiversidad parte de su contexto. Esta perspectiva de enseñanza, debe ser considerada en la parte curricular, con la integración de las diferentes áreas de saberes y conocimientos tanto en el área de Ciencias Naturales, como la indagación. Los estudiantes muestran una actitud positiva y opinión favorable hacia la ciencia, los maestros, maestras y directores muestran interés en la investigación desde la lectura de la realidad, el proceso de aprendizaje con materiales del contexto y laboratorios caseros. Desde esta perspectiva el presente artículo fue elaborado como parte de la experiencia vivida en el transcurso del acompañamiento a los maestros y estudiantes para generar las habilidades lingǘsticas desde el inicio del desarrollo de la capacidad investigativa a través de la realización de algunos experimentos. Visto de esta forma el aprendizaje debe abrir posibilidades más vivenciales para originar propios conceptos. Porque aun en la actualidad, los estudiantes memorizan conceptos, hechos, leyes, fórmulas y ejercicios no se logra desarrollar su espíritu comprensivo, reflexivo e innovador de análisis crítico.
\end{abstract}

\begin{abstract}
The objective of developing research capacity in students is to prepare for the exercise of an active and aware citizenship of biodiversity part of its context. This teaching perspective must be considered in the curricular part, with the integration of the different areas of knowledge and knowledge both in the area of Natural Sciences, as well as inquiry. Students show a positive attitude and favorable opinion towards science, teachers and principals show interest in research from the reading of reality, the learning process with context materials and home laboratories. From this perspective, this article was prepared as part of the experience lived in the course of accompanying teachers and students to generate linguistic skills from the beginning of the development of investigative capacity through the performance of some experiments. Seen in this way, learning should open up more experiential possibilities to originate own concepts. Because even today, students memorize concepts, facts, laws, formulas and exercises, it is not possible to develop their understanding, reflective and innovative spirit of critical analysis.
\end{abstract}

\section{Palabras clave:}

Habilidades; lingüísticas; capacidades investigación y experimentos

\section{Keywords: \\ Virtual strategy; teaching resource; technical drawing}




\section{INTRODUCCIÓN}

$\mathrm{S}$ e inicia esta reflexión a partir de la mirada de la educadora e investigadora argentina Graciela Frigerio (2012), quien expresa, acerca de la vivencia con la palabra y el conocer lo siguiente:

Saber es un verbo asociado a la actividad de educar. Cotidianamente se conjuga en todos los tiempos, modos y personas en los territorios escolares de todas las geografías. Se trata de un verbo acerca de cuyos sentidos no indagamos regularmente, pero que constituye $\mathrm{y}$ deviene en prescripción curricular, contenidos, prácticas, experiencias, efectos. El sustantivo suele ser traducido en contenidos. Imposible sortear el verbo y el sustantivo al tiempo de reflexionar sobre sus alteraciones en los territorios educativos. (p.22)

Para los maestros uno de los principales retos a los que se enfrenta en su día a día, es que el estudiante aprenda a leer y escribir correctamente, ya que la lectura es imprescindible en cualquier área de saberes y conocimientos como en el campo de ciencias naturales, por ejemplo a partir de un experimento se generan procesos de lectura y escritura de textos como: informes, registro de experiencias, la descripción, textos instructivos y otros. La importancia del desarrollo de la investigación se refiere especialmente a los alcances en el proceso de aprendizaje de los estudiantes. De este modo, el proceso educativo debe tomar otro rumbo para mejorar la calidad.

\section{INTERPRETACIÓN DE LA VIVENCIA PEDAGÓGICA}

\section{Desarrollo de las habilidades lingüísticas}

En nuestra práctica habitual no todos hemos desarrollado las habilidades lingüísticas, porque un texto nunca es interpretado de la misma forma por diferentes personas porque, todos tenemos diferentes criterios a la hora de analizar y reflexionar. Por ello, se aborda la siguiente temática con el propósito de fortalecer la problemática identificada, el aprendizaje pasivo en el área de ciencias naturales no permite desarrollar la capacidad investigativa. Por tanto, "debemos decir, que en ello radica como una condición necesaria 
para el aprendizaje, sí lo es para la formación del sujeto. Es decir, podemos hablar de formación toda vez que el sujeto halle sentido en lo que lee y escribe" (Lerner, 2011, p. 120). Uno de los objetivos fundamentales del maestro y en las unidades educativas es insertar la promoción de los niveles de la lectura para la comprensión y los ámbitos de la escritura que van relacionados a través de diversas estrategias en el que el maestro cumple el papel de mediador para consolidar el desarrollo de las habilidades lingüísticas. Tal como se observa que las estrategias son utilizadas con el fin de promover a una comprensión de lectura.

Se visualizan a continuación imágenes de estudiantes de ESFM U-A. Sacaba, en pos del la técnica de la asociación ideas para el desarrollo lingüístico (lectura y escritura). Producción narrativa (cuento, historia y otros).
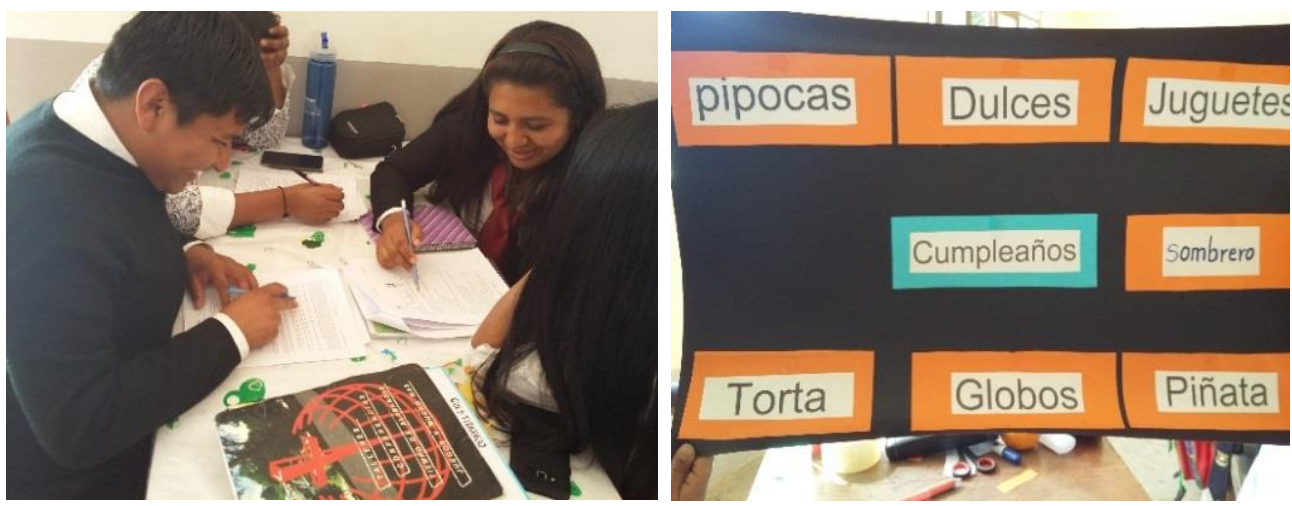

Figura 1. Desarrollo lingüístico. (Fuente: Autoría propia)

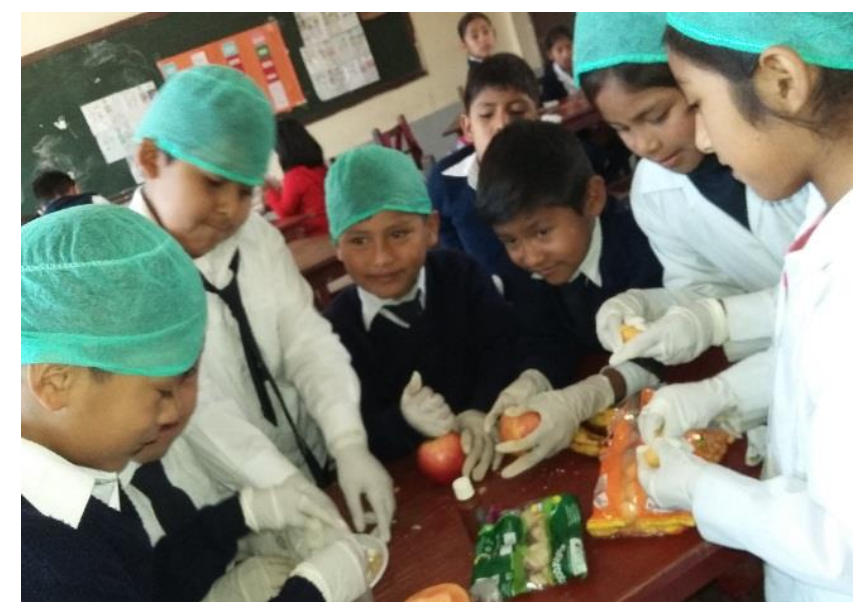

Figura 2.Estudiantes leyendo las envolturas de alimentos 
En esta misma línea, siguiendo a Manzano et al. (2017) "demuestran la importancia del contexto familiar en el desarrollo de habilidades lingüísticas, siendo los padres fundamentales en el proceso de lectura inicial de sus hijos.... El progreso de la esfera lingüística de los niños indica que el desempeño logrado en la edad escolar está en correspondencia con el tipo de crianza en edades tempranas y la estimulación general recibida en el hogar" (pp. 312313). Estas habilidades que se adquieren ayudan a comprender mejor porque los estudiantes, ven avisos comerciales, dibujos, gráficas y saben los nombres de sus dulces favoritos, programas de televisión, letreros que observan en la calle los reconoce por sus colores como (chizitos, pipoca, papa fritas, etc.) son algunas estrategias posibles que ayudan a obtener cambios significativos en relación con la lectura y escritura, ya que desarrollan asertivamente las actividades de experimentos.

\section{Desarrollo de la capacidad investigativa}

La conservación de la biodiversidad es un interés común de toda la humanidad y tiene una importancia crítica para satisfacer sus necesidades básicas. En el Modelo Educativo Sociocomunitario Productivo es el enfoque central del área de ciencia naturales. Al respecto es preciso destacar lo siguiente: "BiocéntricoBiocosmocéntrico, superando desde esta visión, el enfoque antropocéntrico. Asume la visión de las NyPIOs en cuanto la preservación de vida como fundamental, así como las relaciones de respeto, reciprocidad y complementariedad entre los seres vivos". (Programa de Estudio Currículo Base Nivel Primario 2014, p. 35). Tal como se observa el desarrollo de la capacidad investigativa con los estudiantes y profesores. 


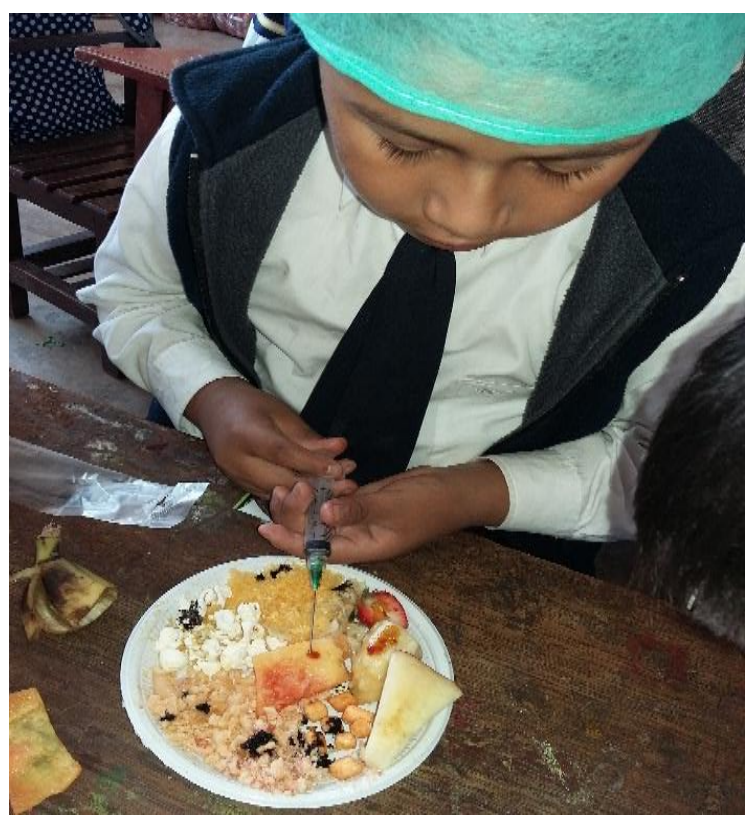

Figura 3. Experimento de la prueba de yodo en alimentos que consume en el recreo
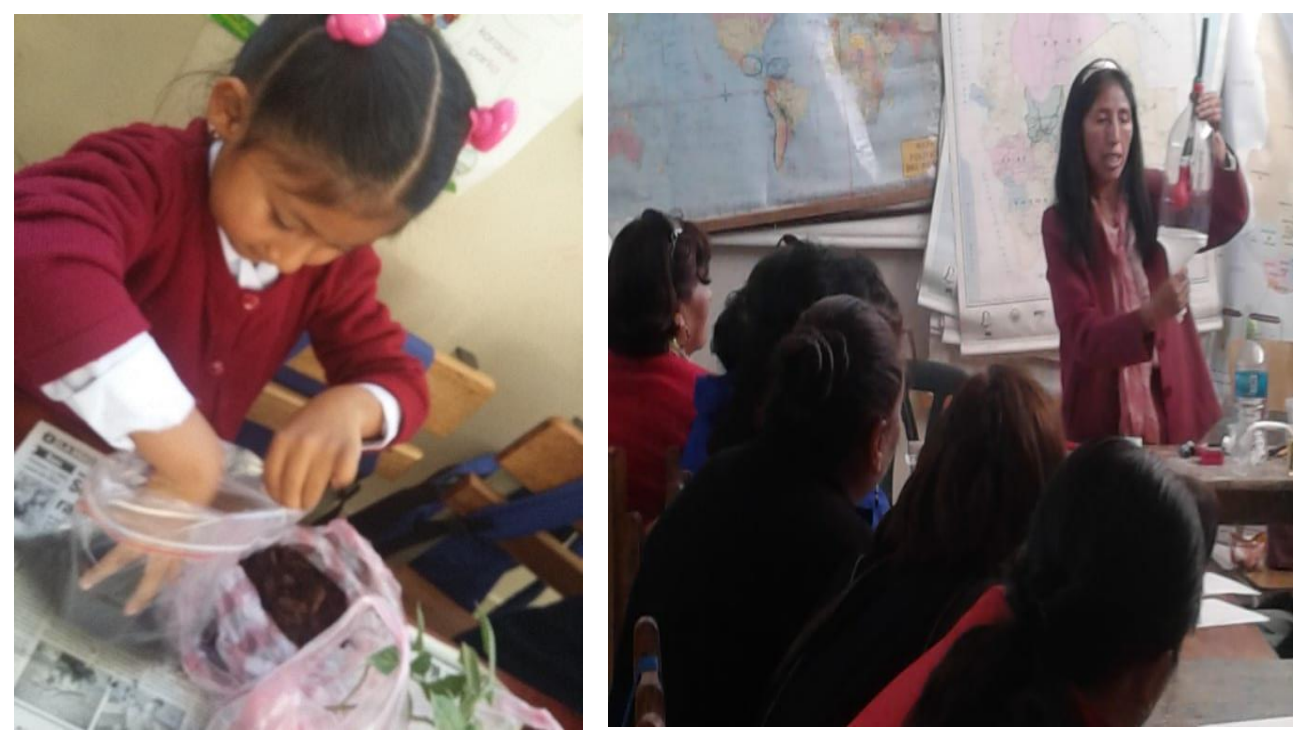

Figura 4. Desarrollo de capacidades investigativas. (Fuente: Autoría propia)

Ahora bien, mucho de lo que los estudiantes aprenden es a través de la observación, la comparación. Algunos ejemplos como los errores de ortografía se debe a que no diferencian entre grafema y fonema, acentuación en las palabras, lo que repercute en las otras áreas de saberes y conocimientos por tanto es fundamental desarrollar la capacidad de observación para encontrar diferencias, semejanzas y otras características de cuanto pueda observar, describir, analizar y reflexionar desde su realidad. Por esto se 
considera importante, trabajar estos aspectos de "aprendizaje de las y los niños desde el énfasis de la Convivencia Biocéntrica Comunitaria se centran en el fortalecimiento de las capacidades, cualidades y potencialidades que serán alcanzadas... en el proceso educativo para conocer y transformar la realidad y apropiarse de los saberes y conocimientos de la humanidad" (Ministerio de educación, 2019. p. 9).

Si bien las posibilidades que se advierten en la Práctica son: a) las experiencias, b) contacto directo con la realidad y c) experimentación. Ante estos cuestionamientos los maestros y maestras manifiestan que el área de ciencias naturales apertura al proceso de aprendizaje observando y comparando, salidas al campo puede ayudar a comprender el área, así como algunos gestores aseveran que los estudiantes expresan al respecto deseos de observar la naturaleza y, desde allí, desarrollar aprendizajes. Esto permite entender la experimentación como una posibilidad de iniciar la clase es poco practicada con los mismos por más insistencia se hace desde las diferentes instancias.

Este nivel de la Práctica hace referencia a las actividades de experimentación propias de ciertos conocimientos científicos. Con esto se enfatiza en que aprendan primero desde la experimentación y luego se reflexione teóricamente sobre el experimento. Hablamos de experimentación en el sentido científico convencional de la palabra: "estos procesos se ponen en acción las capacidades de indagación y experimentación al abordar diferentes contenidos planteados en los programas de estudio". (Ministerio de Educación. 2013, pp.35-36). Llama la atención, sobre la labor del maestro en el proceso educativo, las posibilidades de trabajar en el desarrollo de ciertas capacidades sobre la experimentación, las experiencias son parte de la lectura de la realidad que le ayudaría a comprender en entorno donde vive y protegerla comprendiendo la reciprocidad con la naturaleza y el cuidado del medio ambiente y madurar el pensamiento con la biodiversidad existente.

Desde el aspecto pedagógico es necesario desarrollar la investigación para producir conocimiento a través del Ciclo de Indagación, aplicado a conciencia a través de preguntas que le problematicen, esto se convierte en una herramienta muy poderosa y sofisticada para conocer y entender nuestro mundo y para comprender los acontecimientos que suceden a nuestro alrededor, el Ciclo de Indagación es útil tanto para plantearse hipótesis, un 
proceso de cuestionamiento y sacar sus propias conclusiones dirigidas por el maestro (por ejemplo: la comparación de cuáles plantas crecen en la sombra y cuáles en el sol, porque el agua recorre de abajo hacia arriba y otros), como para entender las relaciones existentes entre el hombre y la naturaleza donde vive, asimismo el uso de una parcelita, como en el patio de la escuela, en la plaza del pueblo, entre otras, que son fuentes de aprendizaje para el estudiante porque le permite explorar, comparar, analizar y plantear su posicionamiento.

Empero, al referirnos acerca de las habilidades lingüísticas (la escucha, el habla, el leer y el escribir), a través de la escritura de textos de informes, ensayos, registro experiencias, textos descriptivos y otros a partir de la experimentación se sistematiza el desarrollo de la capacidad investigativa. Esto tiene que ver con el posicionamiento del maestro y maestra y hacer énfasis en estos aspectos además, que el aprendizaje en el área de ciencias naturales el proceso de formación "Se desarrolla el aprendizaje desde la lectura de textos de apoyo" algunas afirmaciones. (Entrevista. Estudiantes y padres de familia julio 26, 2019).

Este nivel de la Práctica hace referencia a las actividades de experimentación. Se busca que en las mismas aprendan primero desde el proceso y luego reflexionen sobre la investigación. "Hablamos de experimentación en el sentido científico convencional de la palabra... estos procesos se ponen en acción las capacidades de indagación y experimentación al abordar diferentes contenidos planteados en los programas de estudio". (Ministerio de Educación. 2013, pp.35, 36).

Se ahonda, de esta forma, sobre la labor del maestro en el proceso educativo las salidas como las potencialidades, desarrollo de ciertas capacidades de las habilidades lingüísticas sobre la experimentación y la capacidad investigativa son parte de la lectura de la realidad que le ayudaría a comprender en entorno donde vive y protegerla comprendiendo la reciprocidad con la naturaleza, el cuidado del medio ambiente y madurar el pensamiento investigativo con la biodiversidad existente.

No obstante, es perentorio reconocer el nivel de curiosidad que presentan los estudiantes por su medio ambiente y de qué manera los profesores podemos orientar y disfrutar de la experiencia pedagógica que genera explorar múltiples fenómenos naturales. Situaciones de aprendizaje aún se teorizan ya sea por la costumbre 
de la habitual clase pasiva o la comodidad en el proceso educativo que es importante hablar de la investigación.

Como resultado "Esta investigación subraya que nos enfrentamos a problemas en la formación del profesor, que, en su mayoría, son inherentes a otras épocas en las que los recursos didácticos y en Ciencias Naturales eran más deficitarios... con el objetivo de que aprenda a pensar y a tomar conciencia de sus propios procesos y estrategias mentales, consiguiendo una mayor autonomía y mejorando el rendimiento y la eficacia en el aprendizaje. (Araque, 2011). En la medida, de la comprensión de los actores de la educación sobre el aprendizaje del área de ciencias naturales que es necesario identificar los intereses de los estudiantes y que la ciencia debe estar contextualizada y representar una utilidad para los mismos.

Al respecto, de la utilidad de los experimentos en el aprendizaje del área de ciencias naturales no siempre el maestro y la maestra asume como tal, por considerar la inversión de un presupuesto, genera cierta indisciplina con los estudiantes y otros factores. Pero, como decía Freire (2002): el hombre es hombre, y el mundo es mundo, en la medida en que ambos se encuentren en una relación permanente, el mundo será transformado". (Torrez, pág.140) Las expresiones de los estudiantes respecto de la enseñanza de la ciencia están relacionadas con dos aspectos: en primer lugar, la presión de parte de los maestros en responder cuestionarios reelaborados que influye en la mecanización de respuestas desde la lectura de textos apoyo estas situaciones hace en que no desarrollen la capacidad investigativa. No es un secreto los maestros de educación primaria comunitaria vocacional comprenden que la ciencia es un conjunto de conocimientos que permite resolver necesidades, explicar su entorno y principalmente educar a los estudiantes como respuesta a las problemáticas, necesidades y también potencialidad a los cuales el Modelo Educativo Sociocomunitario Productivo intenta mejorar.

El análisis de distintas formas de colaboración de los padres de familia en la escuela, planteada por los maestros y maestras la existencia de por "La escasa participación de los padres en situaciones de aprendizaje de sus hijos" (entrevista a PPFF u.e) sea producto de un reclamo contradictorio, quizás, a las diversas perspectivas, visión y exigencia de los mismos. Aquí es donde se ve casi analógicamente lo que significa "crear un problema; en el fondo es zambullirse más allá de lo observable y para eso hay que contener 
la respiración, que en el caso de la investigación, equivaldría a recurrir al pensamiento crítico, el cual hace las veces de oxígeno. $\mathrm{Si}$ queremos zambullirnos para ver lo que hay debajo del iceberg, debemos tener capacidad de crítica y la capacidad de crítica significa no contentarse con lo que se ve, con lo observable". (Zemelman $\mathrm{H}$. 2015) Desde, el aspecto pedagógico es necesario desarrollar la investigación para producir conocimiento con las habilidades lingüísticas a través de los experimentos y las preguntas que le problematicen al estudiante en al futuro maestro y al mismo maestro en ejercicio que se convierte en una herramienta muy poderosa y sofisticada para conocer, entender y comprender los acontecimientos que suceden a nuestro alrededor.

\section{CONCLUSIONES}

$\mathrm{E}$ $\mathrm{n}$ definitiva, para el maestro la parte fundamental es la concretización del proceso educativo además, de ser consciente de su labor de día a día así mismo, reconocer la debilidad de los estudiantes en cuanto al desarrollo del pensamiento investigativo. A partir de ese reconocimiento de los maestros se toman acciones que les permita comprometerse con el fortalecimiento de la lectura, escritura, área ciencias naturales y otras. Estas particularidades ayudan a la toma de consciencia por parte de toda la comunidad educativa buscando el bien común y que el proceso educativo responda a las necesidades, problemáticas y potencialidades que existe.

Ahora bien, es necesario enfatizar en cuanto a los niveles de comprensión lectora y los ámbitos de la escritura, por ello se presenta algunas puntualizaciones relacionadas con el desarrollo de la lengua oral y escrita, considerando que la comprensión lectora es un proceso de construcción de significado personal del texto mediante la interacción activa con el lector. Al respecto, se consideró lo propuesto por Cassany (2016) en su texto Tras las Líneas. En el fondo se resume para desplegar con énfasis los tres niveles de comprensión lectora: literal, inferencial o deductiva y crítica, tanto los ámbitos de la escritura se podrán trabajar desde el área de ciencias naturales. Ante estos cuestionamientos, los maestros y las maestras manifiestan que el área de ciencias naturales apertura al proceso de aprendizaje observando y comparando y las salidas al campo puede ayudar a comprender la biodiversidad. No obstante, la experimentación 
permite desarrollar el pensamiento investigativo, además una posibilidad de iniciar la clase que muy poco es practicada con los estudiantes.

Partir desde la experiencia se convierte en un elemento estratégico y descolonizador de nuestra metodología..., las teorías no sirven por sí mismas, sólo tienen sentido si expresan una determinada realidad...se puede utilizar situaciones concretas de la vida, observaciones, visitas a espacios productivos...Aquí es importante que las y los estudiantes vean su propia realidad como punto de partida para abordar los contenidos... aprender sobre los elementos de la naturaleza de manera directa, al buscar resolver algún problema real. (Ministerio de Educación. 2013, p.35).

Al mismo tiempo, a manera de cierre, comprender el aprendizaje desde la experimentación, las experiencias que se desarrollan y se fortalecen desde contacto directo con la realidad, llama la atención a propios y extraños con solo observar e identificar elementos importantes de aprendizaje.

\section{REFERENCIAS}

Araque Hontangas, N. (2011) Reflexiones en torno a la enseñanza de las ciencias naturales en las escuelas españolas. Revista Nova scientia. 3 (5) abr. 2011. Guanajuato, México

Cassany, D. (2006).Tras las líneas. Sobre la lectura contemporánea. Anagrama, Barcelona, España

Frigerio, G. (2015). Curioseando (Saberes e ignorancias). Revista Educación y Ciudad, (22), 81-102. Recuperado de https://revistas.idep.edu.co/index.php/educacion-yciudad/article/view/89

Manzano Aragüez, D.; Alemany Arrebola, I. (2017). Análisis de las habilidades lingüísticas en educación infantil y primaria en un contexto multicultural. Profesorado. Revista de Currículum y Formación de Profesorado 21 (2), julio 2017, pp. 309-329. Universidad de Granada. Granada, España

Ministerio de Educación. Unidad de Formación No. 4. "Medios de Enseñanza en el Aprendizaje Comunitario Planificación Curricular". Cuadernos de Formación Continua. Equipo PROFOCOM. La Paz, Bolivia, 2013 
Ministerio de Educación. "Orientaciones para el fortalecimiento de Ciencias Naturales en Educación Primaria Comunitaria Vocacional". Proceso de Acompañamiento a la Implementación del MESCP. La Paz, Bolivia, 2019

Sheckles, Mary. Cómo enseñar las Ciencias al escolar. Editorial Paidos. Argentina, 1964

Tacca Huamán, Daniel Rubén. La enseñanza de las Ciencias Naturales en la educación básica. Revista Investigación Educativa, Vol. 14 N.ㅇ 26, 139-152. Julio-Diciembre 2010. Lima, Perú, 2010

Torres Salas, M. La enseñanza tradicional de las ciencias versus las nuevas tendencias educativas. Revista Electrónica Educare, vol. XIV, núm. 1, enero-junio, 2010, pp. 131-142. Universidad Nacional. Heredia, Costa Rica, 2010

Zemelman, H. (2001, noviembre). Pensar teórico y pensar epistémico: los retos de las ciencias sociales latinoamericanas. En Galeana 96. México 